\title{
Tensile Properties of Treated and Untreated Paddy Straw Fiber Using Sodium Hydroxide Strengthened with Polypropylene Resin
}

\author{
Masni A. Majid ${ }^{1, a}$, Mimi Attahirah Mohd Hishammudin ${ }^{1}$, Noor Azlina Abd Hamid ${ }^{1}$, Zalipah Jamellodin ${ }^{1}$ \\ and Norhafizah Salleh ${ }^{1}$ \\ ${ }^{1}$ Faculty of Civil and Environmental Engineering, Universiti Tun Hussein Onn Malaysia, 86400 Parit Raja, Johor, \\ Malaysia
}

\begin{abstract}
This paper focus on the effect of alkaline treatment by using sodium hydroxide on the tensile properties of paddy straw fibers strengthened with polypropylene resin. Two types of paddy straw fibers were used which are MR219 and MR220. The paddy straw fibers were prepared in two conditions as untreated and treated with sodium hydroxide $(\mathrm{NaOH})$ at $5 \%$ for 24 hours and then dried at $80^{\circ} \mathrm{C}$ for another 24 hours. For sampling process, these fibers were weighted according to $5 \%$ and $10 \%$ mass fraction. A total of 16 samples were prepared for this study based on ASTM D638 and tensile test was conducted by using ASTM D5083. The result showed that paddy straw fiber treated with $\mathrm{NaOH}$ had gain higher ultimate tensile strength compared to untreated paddy straw fiber where the highest ultimate tensile strength for the fibers is recorded at $10 \%$ of MR219 fiber with value of $2.0230 \mathrm{kN}$ and $3.677 \mathrm{~mm}$ displacement were recorded. The highest strain were recorded by the same fibers with an average value of $5.253 \%$ and obtained the Young's modulus up to $1110 \mathrm{MPa}$. However, the Young's modulus which has been obtained by the same fibers was decreased with the percentage difference of $40 \%$.
\end{abstract}

\section{Introduction}

The use of synthetic fibers can cause pollution to environment and increment of $\mathrm{CO}_{2}$ that contribute to effect of global warming [1]. Therefore, researchers nowadays attempted to make changes to the materials that are harmful to humans and environment by finding sustainable and environmental friendly material as an alternative in replacing synthetic fibers.

Natural fibers have the potential to be used as reinforcement in engineering application [2]. Natural fiber is a material that is derived from its own natural resources resulting from plants and animals. There are several types of traditional materials are used as reinforcing material such as wood and steel. Based on previous researcher's studies, natural fibers has advantages of low cost, environmental friendly, renewable, low density, biodegradability and has satisfactory mechanical properties [3]. At present, research is carried out to make the plant natural fibers used as reinforcement fibers replacing iron. In a recent study, composite was produced with the combination of resin to improve the mechanical properties of tensile, impact and flexural properties [4].

\footnotetext{
a Corresponding author : masni@uthm.edu.my
} 
Many researchers have conducted study on the mechanical properties of natural fiber composites using polymers matrix. The polymer matrix or binding agent has ductile more than fibers and it acts as a strengthening of composite. Furthermore, it also serves as to protect the fiber from environmental damage before, during and after the composition process [5]. Among the natural fibers used in previous research are paddy fiber [1, 6], kenaf [2, 4, 7, 8], bamboo [9], abaca [3] and others. Thus this study was proposed to investigate the effect of alkaline treatment on tensile properties of paddy straw fiber.

\section{Materials and Methods}

\subsection{Material preparation}

Paddy straw raw fibers used in this study was supplied from paddy industry in Selangor. The supplied fibers were hand-washed, and dried at temperature of $70^{\circ} \mathrm{C}$ for 24 hours as shown in Figure 1. The sodium hydroxide $(\mathrm{NaOH})$ solution used for fiber treatment and the polymer used for binding agent were polypropylene (PP).

\subsection{Fiber treatment}

In this study, alkaline treatment has been applied to the paddy straw fibers. Figure 2 shows the fibers were soaked in $5 \% \mathrm{NaOH}$ solution for 24 hours. The fibers were then washed several times with distilled water to remove the excess of $\mathrm{NaOH}$ and dried in an oven at $80^{\circ} \mathrm{C}$ for 24 hours.

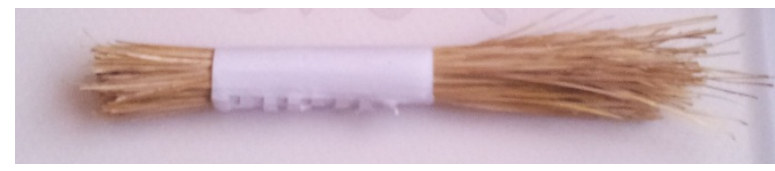

Figure 1. Paddy straw fiber.

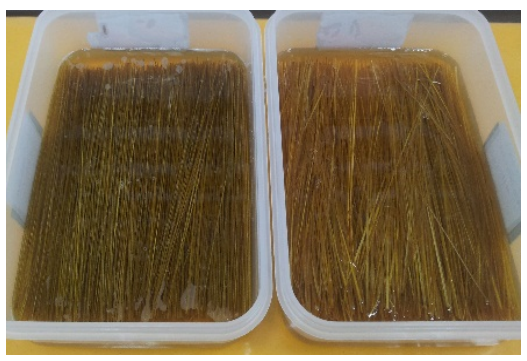

Figure 2. Treatment of paddy straw fiber.

\subsection{Sample preparation}

After alkaline treatment process, the paddy straw fibers and the polypropylene were weighted according to the ratio of (1:9) and (0.5:9.5) for each specimen. In the sampling process, a metal mould $\left(100 \times 20 \times 6 \mathrm{~mm}^{3}\right)$ was coated with a layer of release agent (WD-40). The treated fibers were arranged into the mould using sandwich method. Finally, the composite was covered with mould cover and compressed by using Hot Press Machine at $230^{\circ} \mathrm{C}$ for 20 minutes as can be seen in Figure 3. The same procedure was done for untreated paddy straw fibers as well. The specimen set has been produced for testing purposes as shown in Table 1. 


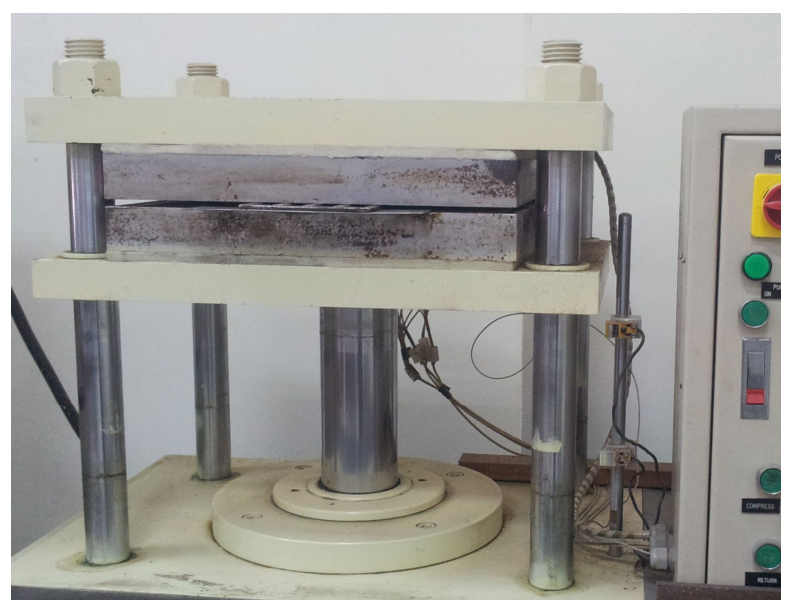

Figure 3. Compression process.

Table 1. Information on specimens produced for testing.

\begin{tabular}{ccl}
\hline Specimen No & Paddy Fiber Type & \multicolumn{1}{c}{ Resin } \\
\hline $\mathbf{1}$ & Untreated MR219 fibers & Polypropylene \\
$\mathbf{2}$ & Treated MR219 fibers & Polypropylene \\
$\mathbf{3}$ & Untreated MR220 fibers & Polypropylene \\
$\mathbf{4}$ & Treated MR220 fibers & Polypropylene \\
\hline
\end{tabular}

\subsection{Tensile test}

In a broad sense, tensile test is measuring the ability to withstand forces; tend to pull it apart and to what extent the material stretches before breaking. Universal Testing Machine was used at cross-head speed of $1 \mathrm{~m} / \mathrm{min}$. The ultimate tensile strength was obtained from the maximum stress on the stressstrain curve. Besides ultimate tensile strength, the strain at break was also obtained from the stressstrain curve. The modulus elasticity was provided as the slope of the linear portion of stress-strain curve. Three specimens for each case were tested for treated fibers and three specimens for each case tested for untreated fibers as a control. The specimens were pulled until it fractured.

\section{Results and Discussion}

\subsection{Tensile strength}

Figure 4 presents the maximum ultimate tensile strength of alkaline treatment and untreated paddy straw fiber composites. For MR220 type of paddy fiber, the impact load is slightly small on both composites because it cannot accommodate higher load. However, the MR219 type of paddy fiber gave higher value of ultimate tensile strength compared with MR220.

Based on the histogram, it is obvious that $10 \%$ mass fraction of fibers gave higher value of ultimate tensile strength compared to 5\% mass fraction of fibers for MR219 fibers and opposite result for MR220 fibers. For MR219 fibers, an approximation of 40\% and 29\% increment is noticed for both treated and untreated cases respectively. As for MR220 fibers an approximation of 16\% and 26\% decrement recorded for treated and untreated case respectively. It is worth nothing that the treated of 


\section{MATEC Web of Conferences}

paddy straw fiber with $\mathrm{NaOH}$ solution influences the tensile properties of composites. In particular, the treated fiber of MR219 in composite of 5\% mass fraction show ultimate tensile strength of 31\% higher than untreated fiber of MR219 in the same composite of mass fraction. It is explained by the increase in uniformity of the yields to enhance strength due to the removal of impurities during treatment. On the other hand, the chemical treatment of paddy fibers leads to increments of $42 \%$ in tensile strength of the $10 \%$ fibers mass fraction composites.

Moreover, the treated fiber of MR220 also experienced higher ultimate strength of $9 \%$ and $21 \%$ respectively for both $5 \%$ and $10 \%$ mass of fractions. However, the MR220 obtained lower ultimate strength value compared to MR219 in 10\% mass fractions. Based on research [2], noted that the high impact strength will cause a lower ultimate tensile strength achieved. The result of both type of paddy fibers showed that the $5 \% \mathrm{NaOH}$ treatment is an evident that the alkali treatment helps in improving the tensile strength and chemical bonding between fibers and resin. It enhances surface adhesion characteristics of fibers by removing impurities and produce rougher fiber surface.

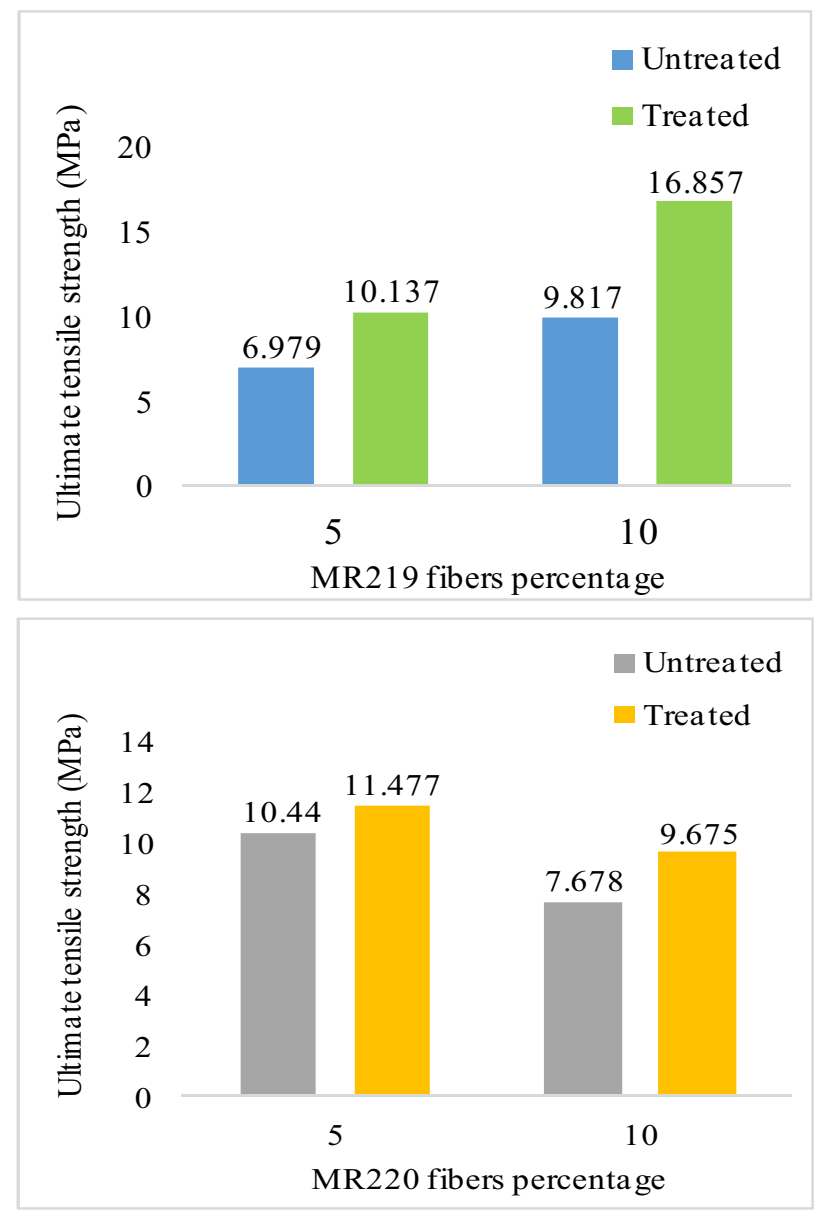

Figure 4. Ultimate tensile strength.

\subsection{Modulus elasticity}

Based on the graph plotted in Figure 5, the modulus of elasticity was decreases between treated fibers with untreated fibers. This result obtained as opposed to the result recorded by previous researcher [3] using abaca fibers. 

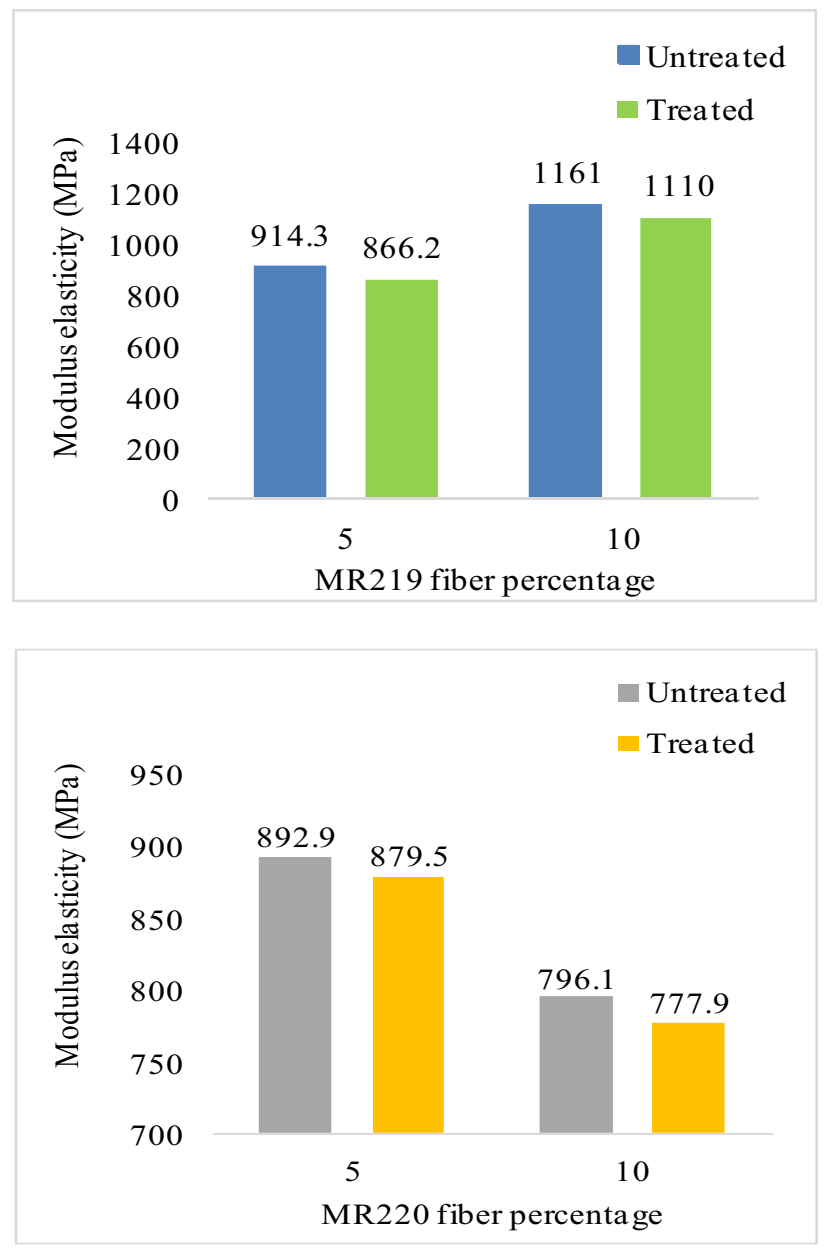

Figure 5. Modulus elasticity.

The values of modulus elasticity recorded are uncertain due to the immersion time, arrangements of fibers are not aligning properly and also the thickness is not the same along the length of specimen. A few researcher reported that this scenario occurred due to longer immersion time that had detrimental effect on the fibers surface and consequently on their mechanical properties [3, 4]. Generally, the existence of alkalization treatment on specimens related to fibers contributed to the value of modulus elasticity.

\section{Conclusions}

The paddy straw fibers is one of the environmentally friendly natural fiber, easily available, renewable resources, recyclable, widely distributed, good acoustic insulation and economy compared to carbon and glass fibers. In this research, the effect of alkaline treatment $(5 \% \mathrm{NaOH}$ solution) on tensile properties of paddy straw fibers composites was evaluated and it can be summarized that: (i) $\mathrm{NaOH}$ produced a better properties compared to the untreated fibers as the $\mathrm{NaOH}$ treatment has highly enhanced the interfacial adhesion of the fiber with the resin. MR 219 produce $31 \%$ and $42 \%$ increment from 5\% and 10\% mass of treated fiber compared to untreated fiber, while MR220 produce $9 \%$ and $21 \%$ increment respectively, (ii) The $10 \%$ mass of treated paddy fibers produce higher 
ultimate tensile strength as compared to $5 \%$ mass of treated paddy fiber with the difference of $40 \%$ increment for MR219 fibers and opposite result for MR220 fibers with 16\% decrement, and (iii) The modulus elasticity of treated paddy straw fiber decreased compared to untreated paddy straw fibers. The fibers surface and their mechanical properties effect during the immersion time.

Overall, it can be concluded that the $10 \%$ treated MR219 paddy fibers composite gave the highest ultimate tensile strength while the lowest ultimate strength was obtained from 5\% untreated MR219 paddy fibers composite. There is a possibility of replacing the synthetic fibers with paddy fibers for tensile applications.

\section{Acknowledgment}

The authors wish to thank Universiti Tun Hussein Onn Malaysia (UTHM) for the financial support and not to forget, technical officer at Faculty of Civil and Environmental Engineering, UTHM for their helps in order to complete this project.

\section{References}

[1] Y. Abdullah, A. Putra, H. Efendy, W.M. Farid and M.R. Ayob, Investigation on sound absorption coefficient of natural paddy fibers. Int. J. of Renewable Energy Resource, 3, 8-11, (2013).

[2] M. Mohd Suhairil, O. Muhammad Fauzi, H. Hazran, R. Muhammad Fairuz, M.S. Mohd Syahar, Improving tensile properties of kenaf fibers treated with sodium hydroxide, Procedia Engineering 41, 1587-1592, (2012).

[3] M. Cai, H. Takagi, A.N. Nakagaito, M. Katoh, T.U. Geoffrey, I.N. Waterhouse and Y. Li, Influence of alkali treatment on internal microstructure and tensile properties of abaca fibers, Industrial Crops and Products, 65, 27-35, (2015).

[4] V. Fiore, G. Di Bella and A. Valenza, The effect of alkaline treatment on mechanical properties of kenaf fiber and their epoxy composites, Composites: Part B, 68, 14-21, (2015).

[5] S. Taj, M. Munawar Ali and Shafiullah Khan, Natural fiber-reinforced polymer composites, Proc. Pakistan Academic Science, 44(2), 129-144, (2007).

[6] R.M. Nasir and N.M. Ghazali, Tribological performance of paddy straw reinforced polypropylene (PSRP) and unidirectional glass-pultruded-kenaf (UGPK) composites. J. Tribologi, 1-17, (2014).

[7] H.M. Akil, M.F. Omar, A.A.M. Mazuki, S. Safiee and Z.A. Mohd Ishak and A. Abu Bakar, Kenaf fiber reinforced composites: A review, Material and Design, 32, 4107-4121, (2011).

[8] M. Edeerozey,Chemical Modification of Kenaf Fibers, Material Letters, 61, 2023-2025, (2007).

[9] K. Murali Mohan Rao, Extraction and tensile properties of natural fibers: Vakka, date and bamboo, Composite Structures 77, 288-295, (2007).

[10]M. Ali, Natural fibres as construction materials, J. of Civil Engineering and Construction Technology, 3(3), 80-89, (2012).

[11] S.N. Monteiro, Natural-fiber polymer-matrix composites: Cheaper, tougher, and environmentally friendly, Journal of the Minerals, Metals and Materials Society, 61(1), 17-22, (2009).

[12]N. Sgriccia, Characterization of natural fiber surfaces and natural fiber composites, Journal of Composites: Part A, 39, 1632-1637, (2008). 